\title{
Why Krogdahl's Flat Space-Time Cosmology Is Superior to General Relativity
}

\author{
Eugene Terry Tatum \\ 760 Campbell Ln., Bowling Green, KY, USA \\ Email: ett@twc.com
}

How to cite this paper: Tatum, E.T. (2017) Why Krogdahl's Flat Space-Time Cosmology Is Superior to General Relativity. Journal of Modern Physics, 8, 2087-2095. https://doi.org/10.4236/jmp.2017.813127

Received: October 24, 2017

Accepted: December 2, 2017

Published: December 5, 2017

Copyright $\odot 2017$ by author and Scientific Research Publishing Inc. This work is licensed under the Creative Commons Attribution International License (CC BY 4.0).

http://creativecommons.org/licenses/by/4.0/

\section{(c) (i) Open Access}

\begin{abstract}
This paper briefly discusses existing problems with the theory of general relativity despite remarkable accuracy in most of its applications. The primary focus is on existing problems in the field of cosmology, particularly those pertaining to expectations of global cosmic space-time curvature in the absence of observational proof. The discussion centers on Krogdahl's recent Lorentzinvariant flat space-time cosmology and its superiority to general relativity with respect to accounting for global cosmic space-time flatness and dark energy observations. The "cosmological constant problem" is briefly addressed as a problem for general relativity with respect to particle physics and quantum field theory. Finally, two very specific validation predictions in favor of Krogdahl's flat space-time cosmology are made with respect to ongoing studies, including the dark energy survey (DES).
\end{abstract}

\section{Keywords}

Cosmological Theory, General Relativity, Flatness Problem, Cosmic Inflation, Dark Energy Survey, Cosmological Constant Problem, Krogdahl's Relativity

\section{Introduction and Background}

The theory of general relativity is an improvement on Newton's gravitational theory by virtue of the fact that its complex differential geometry more accurately passes certain crucial canonical tests of gravitation [1].

Canonical Tests of Gravitation

1) Mercury's advance of perihelion

2) Solar light bending

3) Gravitational redshift

4) Echo delay of sun-grazing light beams

5) Gravity waves 
6) Extreme mass densities of galactic centers

7) A mathematical model of dark energy observations

As a result, it is current scientific belief that four-dimensional space-time must be curved wherever there is a concentration of mass-energy. Thus, it is widely accepted that a cosmological model must have a global space-time curvature which varies in magnitude, and possibly even sign, over the course of cosmological time. And, although flat space-time Lorentz-invariant models of gravitation and cosmology have been attempted from time-to-time over the previous century, they have all failed to pass these crucial gravitational tests.

Current Problems with General Relativity

a) The Schwarzschild Metric and the Infinite Singularity

There are a few nagging doubts as to general relativity's mathematical accuracy in extreme high mass-energy density conditions. In the Schwarzschild metric, for instance, the term $\left(1-2 \mathrm{GM} / \mathrm{c}^{2} \mathrm{r}\right)$ within one of the denominators leads to a seemingly nonsensical result should the astrophysical (or cosmological) body have a radius $\mathrm{r}$ equal to $2 \mathrm{GM} / \mathrm{c}^{2}$. Because mathematicians abhor division by zero, even Einstein doubted that Schwarzschild's "singularity" discovery had any real physical meaning. Contemporary physicists and cosmologists argue vehemently as to whether a true singularity condition of infinite mass-energy density could exist, whether the singularity be astrophysical or cosmological in nature. In addition, Penrose [2] and Hawking have successfully argued that astrophysical and cosmological singularities, if they could exist, would have to have certain identical features. Furthermore, quantum experts have used the uncertainty principle to support the idea that infinite mass-energy density would clearly violate quantum mechanics. This is a fundamental principle of loop quantum gravity theorists, who vigorously dispute the possibility of an infinite singularity.

b) Incompatibility with Quantum Mechanics

The general relativity singularity is but one of many inconsistencies between general relativity and quantum mechanics, heretofore the most successful branch of physics. A quantum theory of gravity which harmonizes general relativity with quantum mechanics seems elusive, and perhaps for good reason. It is generally agreed that the problem with this incompatibility must lie within general relativity. On the other hand, special relativity, with its Lorentz invariance, has already been successfully integrated into quantum field theories of the electromagnetic, weak and strong nuclear forces.

c) Applications to Cosmology, Including the Flatness Problem and the Cosmological Constant Problem

While general relativity appears to accurately model local gravitational effects, there appear to be significant problems in its application to cosmology. Not the least of these is the global cosmic space-time "flatness problem." Cosmologists first became aware of this problem in the late 60's after physicist Robert Dicke made note of it [3]. This concerns the fact that astronomical observations to date show no evidence whatsoever of global cosmic space-time curvature. Recent highly detailed astronomical studies [4] show minimal anisotropy of the cosmic 
microwave background radiation (CMBR) to within about 1 part in 100,000. So, observational studies going all the way back to the cosmic fireball show evidence of extreme global cosmic space-time flatness. If we think of this in terms of a ballistic model of cosmic expansion, the expansion since the time of the cosmic fireball appears to have been very nearly, or exactly, at the gravitational escape velocity required according to our best estimate of total cosmic mass-energy density. As Dicke first pointed out, this would be an extraordinary coincidence for a cosmic model behaving strictly according to general relativity. Furthermore, since 1998, Type Ia supernovae studies have shown our cosmic expansion to be either coasting at constant velocity [5] [6] [7] [8] or slightly accelerating [9] [10] [11]. One thing is certain: the expansion is clearly not decelerating. This discovery alone was worthy of the 2011 Nobel Prize.

However, if general relativity is to be maintained, for a cosmic expansion which does not decelerate, the field equations now require an extremely small but non-zero cosmological constant. And, to make matters worse, a non-zero cosmological constant, according to particle physicists and quantum field theorists, would appear to require an extremely high energy density within the vacuum of space, if it is to be accounted for by virtual particles. If this is to be believed, such high energy density in the cosmic vacuum should imply (at least according to the curved space-time dogma of general relativity) an extreme degree of observable global cosmic space-time curvature. This "cosmological constant problem" [12] [13] is often described as the most embarrassing and vexing problem in all of physics. In contrast, Krogdahl's relativity has no requirement for a cosmological constant or a non-zero energy density within the cosmic vacuum.

\section{Krogdahl's Cosmology of Flat Space-Time and Implications Concerning These Current Problems}

\section{a) Passing the Canonical Tests of Gravitation}

Without belaboring the point, the time has come to search for a better theory of gravitation which not only passes all of the conventional tests of gravitation but also those pertaining to observational cosmology. After recently developing a highly accurate heuristic model of flat space-time cosmology [14] [15], this author began a search for Lorentz-invariant cosmological models which meet these criteria. To his pleasant surprise, he found a little-noticed paper written by physicist W.S. Krogdahl, a general relativity expert, which was published on arXiv.org in final form in 2006 under the title, "Cosmology in Flat Space-Time" [1]. This remarkable paper develops a Lorentz-invariant cosmology (with inspiration from Milne's kinematic relativity approach) that is clearly the path that Einstein should have taken following his development of special relativity. It is well-known that Einstein treated gravitation as equivalent to acceleration. Krogdahl, on the other hand, simply used special relativity's mass-energy equivalence. He treated gravitational work done, and thus energy acquired (during gravitational attraction), in Newtonian gravitational work functions, as an in- 
crease of mass. Mass cannot be regarded as fixed during gravitational interactions! The resulting modified classical equations are then converted into an energy-momentum 4-vector and then conservation of energy equations are converted into Lagrange's equations. The equations which follow this approach very accurately solve every one of the tests of gravitation listed at the beginning of this paper. The reader is referred to Krogdahl's paper (his pages 12-25) for the details.

The most interesting result of Krogdahl's Lorentz-invariant cosmology is that it is a highly accurate theory of gravitation in flat space-time. Einstein's equivalence principle had led him into the complex differential geometry of a curved space-time, to replace the idea that gravity is a force. Krogdahl's approach, by virtue of the fact that gravitational mass is the ratio of gravitational force to gravitational acceleration (Newton's second law) simply flipped Einstein's equivalence on its head and achieved a far simpler geometry by inserting mass-energy equivalence into Newtonian force and work functions.

If one can accept the fact that heuristic [14] [15] and Lorentz-invariant [1] [16] cosmological models of flat space-time are now highly accurate, we can address how existing cosmological problems under general relativity can be resolved.

b) Flat Space-Time Solves the Cosmic Flatness Problem

The cosmic inflation theory was developed primarily to address Dicke's "flatness problem" [17]. The primary reason why Planck survey observations [4] of the CMBR are supposedly supportive of the current theory of cosmic inflation is the extreme observational flatness (minimal anisotropy) of the CMBR. However, a perpetually flat space-time cosmological model does not require the yet-undiscovered new physics (the "inflaton" field, for instance) of cosmic inflation. A theory of cosmic inflation would appear to become unnecessary.

c) Krogdahl's Luminosity Attenuation Factor and an Explanation for Dark Energy Observations

A perpetually flat space-time cosmic model also solves the problem of explaining the dark energy observations of Type Ia supernovae. As mentioned in Krogdahl's paper (his pages 31-33), particularly fast-moving (i.e., more remote) Type Ia supernovae would be expected to exhibit “...ever-diminishing increments of visible starlight because of the increase in redshift with distance..." Even more importantly, however, and for the primary reason Olber's paradox is now solved, fast-moving supernovae would be expected to be remarkably attenuated. To quote Krogdahl, in flat space-time, this “... attenuation must be allowed for, else the calculated distance will be greater than the true distance by a factor of $\left[\left(s^{2}+1\right) / 2 s\right]^{1 / 2}$. This same effect is diminishing the apparent brightness of the Type Ia supernovae ... Uncorrected distances are then interpreted to imply accelerating expansion of the universe. Presumably, the corrected distance would not show this artificial acceleration."

This author couldn't agree more with Krogdahl's concern regarding the Type Ia supernovae observations. Extraordinary claims require extraordinary evi- 
dence. There are now a number of papers [5] [6] [7] [8] showing analysis of the accumulated Type Ia supernovae data which convincingly refutes the idea that dark energy cosmic acceleration is proven. This author would like to see a more rigorous analysis of these data, taking into account the potential for luminosity attenuation errors of the type mentioned above. Crucially, observational distances must be recalculated (i.e., corrected) according to Krogdahl's flat spacetime luminosity attenuation factor of $\left[\left(s^{2}+1\right) / 2 s\right]^{1 / 2}$ and not by different equations derived from general relativity. There may be a Nobel Prize awaiting the graduate student who conducts such a recalculation, particularly if, as expected, it successfully refutes cosmic acceleration.

While the type Ia supernovae data appear to be sufficient to rule out evidence of cosmic deceleration, the data do not at all appear to rule out cosmic expansion at constant velocity, which would be expected for a globally flat universe following Krogdahl's development. The following open source graphs (Figure 1 and Figure 2) from the Supernova Cosmology Project [18] [19] are provided as proof.

In Figure 1, the curve labelled "without vacuum energy" is the curve predicted by the supernova teams prior to their discovery of dark energy. The straight line which separates the pink-shaded part of the graph (representing a decelerating universe) and the blue-shaded part of the graph (representing an accelerating universe) is where the heuristic flat space-time cosmology model and Krogdahl's model fall. This is a flat, constant velocity, universe line. This open source graph identifies this flat universe line as "empty" (i.e., devoid of matter), presumably because Krogdahl's relativistic but globally flat space-time

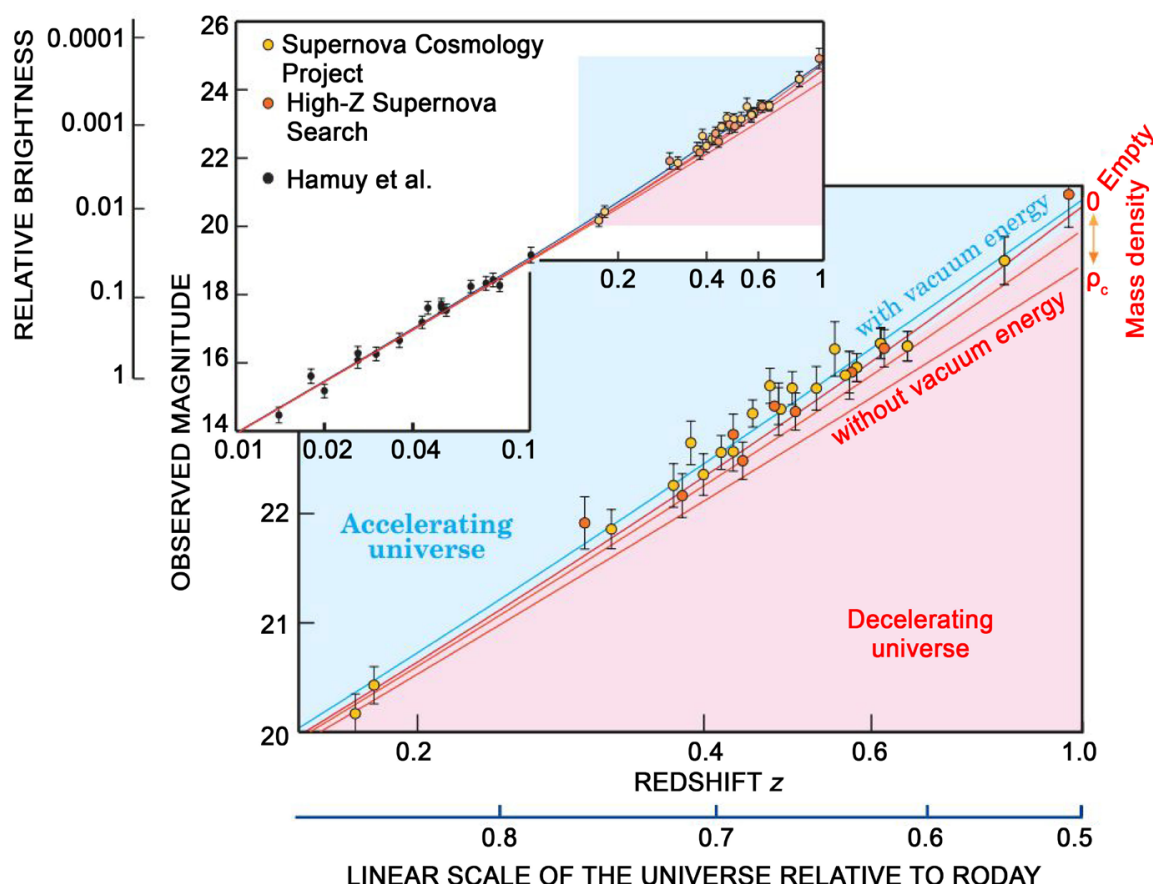

Figure 1. Accumulated Type 1a supernovae data showing observed magnitude as a function of Redshift $z$. 


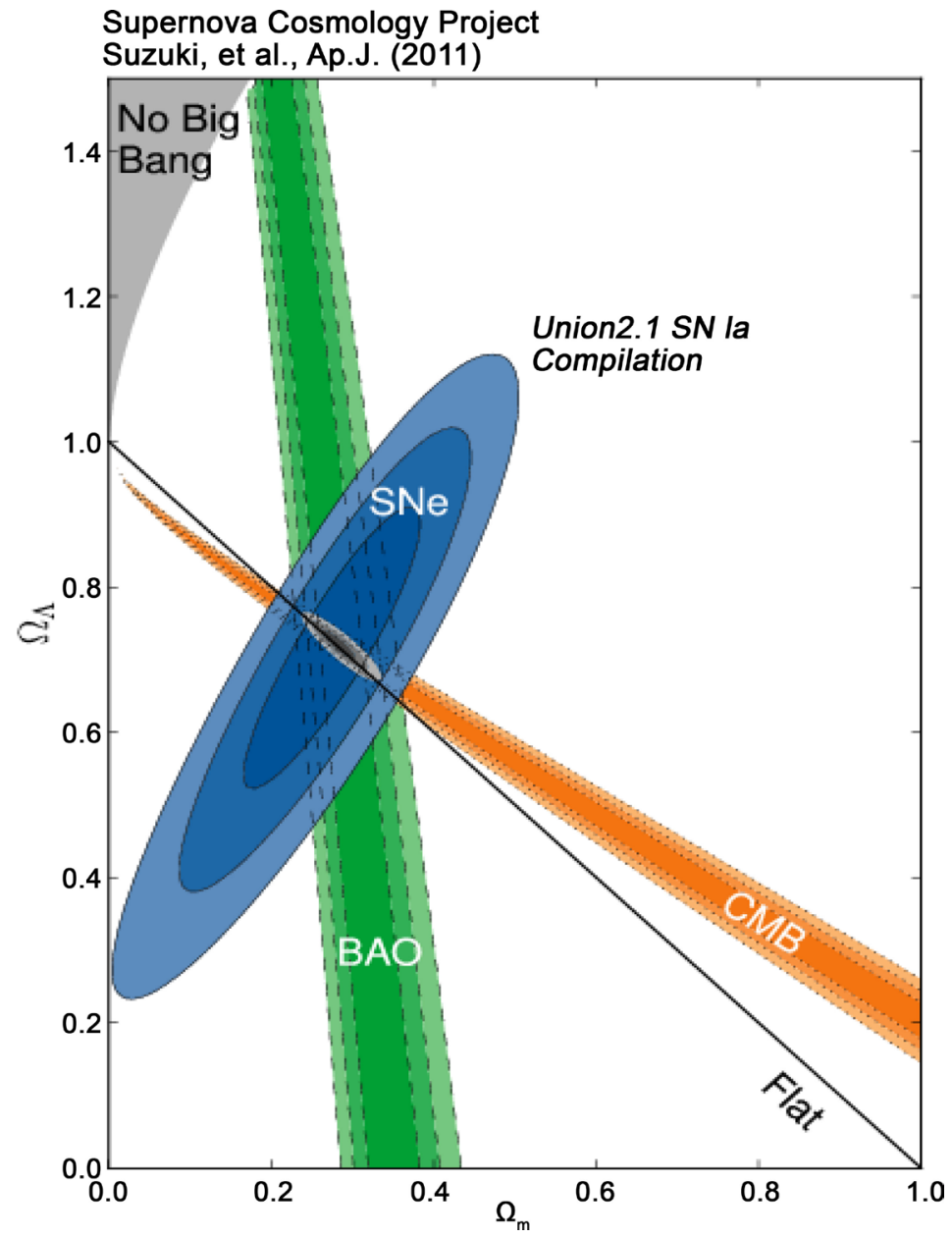

Figure 2. Accumulated supernovae ( $\mathrm{SNe}$ ), barionic acoustic oscillation (BAO), and cosmic microwave background $(\mathrm{CMB})$ constraints on cosmic energy density distribution, reported in the 2011 Supernova Cosmology Project compilation report authored by Suzuki, et.al. [19].

model of cosmology was unknown to most cosmologists and astrophysicists at the time (2011).

In Figure 2, notice how the "flat" cosmic model line passes through the highly constrained shaded best fit zone for all three data sets. It is quite obvious in these open source graphs from the Supernova Cosmology Project that acceptable globally flat space-time cosmology models would be a perfect fit for the accumulated data so far.

Here it is useful to note that, although cosmological redshift is evidence of cosmic expansion, it is not an observational discriminator between the "stretching" space-time of general relativity and the Lorentz-invariant relativistic Doppler redshift (stretching wavelength) of flat space-time cosmology. Both cosmologies give entirely different explanations for the redshift, but rely on the same relativistic formula, $s=(z+1)=[(1+v / c) /(1-v / c)]^{1 / 2}$. This formula was used successfully in this author's first two papers on the heuristic "Flat Space Cosmology" (FSC) model [14] [15]. 
If it is not attributable to cosmic acceleration, how then do we account for dark energy? The observable universe appears to be at, or very near, critical density [4]. An estimated 70\% of this universal total mass-energy density initially appears to be unaccounted for (i.e., "dark"), even taking into account the fact that there appears to be an observable ratio of approximately 5.5 parts dark matter (whatever this turns out to be) to 1 part visible baryonic matter. Even if cosmic acceleration does not account for this dark energy, any flat space-time (i.e., non-accelerating) model is still required to account for it in some way, in order to explain Hubble parameter estimates corresponding to cosmic critical density.

With some simplifying assumptions, Krogdahl cleverly accounts for dark energy as the estimated sum total of gravitational potential mass-energy within the cosmic system. Krogdahl's calculated ratio is 18 parts of this "dark" gravitational potential mass-energy to 1 part visible baryonic matter mass-energy (his page 25). This suggests a cosmic mass-energy distribution of approximately $4 \%$ visible baryonic matter, $24 \%$ dark matter, and $72 \%$ dark energy. This approximation result is very close to current observational estimates [4].

\section{Two Validation Predictions of Krogdahl's Model}

a) Improved Estimations of "Dark" Gravitational Potential Mass-Energy Are Expected to Match the Calculated Percentage of Dark Energy in the Cosmic System

A similar but deeper calculation (by a supercomputer) of the total cosmic gravitational potential mass-energy is now in order. This could also be a Nobelworthy study for a graduate student. As already suggested by Krogdahl's simplified estimate, a more-refined mass-energy estimate is predicted to correlate very closely to what is currently being called dark energy. If this proves to be the case, the mass-energy density distribution of the cosmic system would be entirely accounted for and closely approximate current observational estimates of an expanding cosmic system at critical density, but crucially, without a cosmic acceleration energy component.

b) Luminosity Attenuation Factor Corrections of Supernovae Observational Distances Should Refute Cosmic Acceleration

A rigorous re-analysis of the accumulated Type Ia supernovae data, incorporating Krogdahl's flat space-time luminosity attenuation factor of $\left[\left(s^{2}+1\right) / 2 s\right]^{1 / 2}$ for distance correction, is expected to put cosmic acceleration in further doubt. A predicted graph of redshift $\mathrm{z}$ as a function of cosmic observational distance, properly corrected according to Krogdahl's model, is shown in Figure 3.

A graph of similar shape was shown in Figure 2 of the seminal paper of the heuristic flat space-time cosmology model [14]. Crucially, an accelerating cosmic expansion would demonstrate a flatter curve in such a graph. This effect would become progressively more noticeable at greater observational distances. The pending final results of the dark energy survey (DES), properly corrected for luminosity attenuation and plotted on such a graph as presented in Figure 3, are 


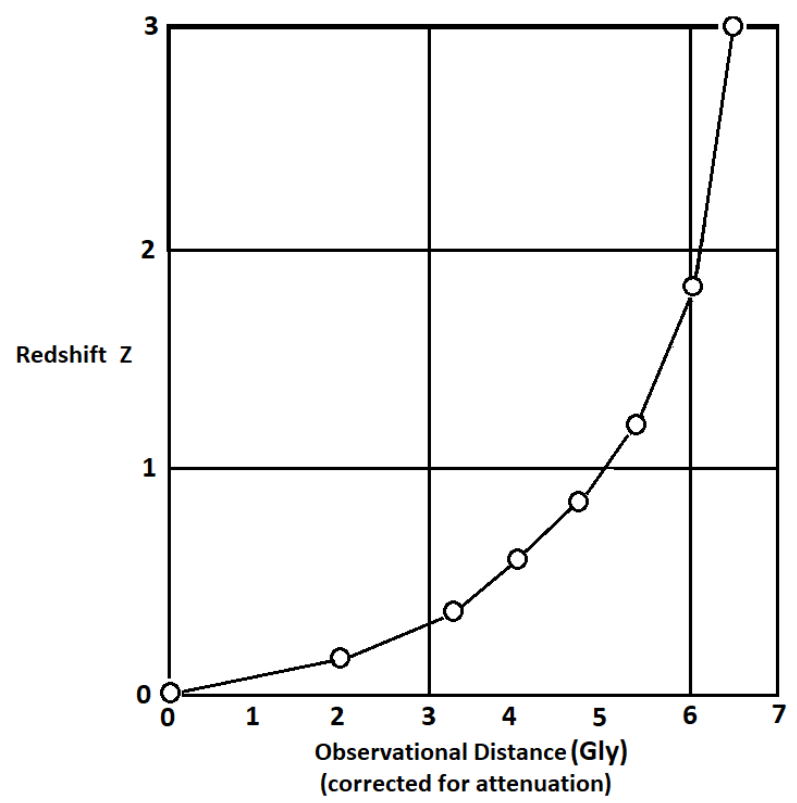

Figure 3. This shows redshift $\mathrm{z}$ of Type 1a supernovae as a function of corrected observational distance (in billion light-year units) calculated according to Krogdahl's luminosity attenuation factor of $\left[\left(s^{2}+1\right) / 2 s\right]^{1 / 2}$.

predicted by this author to show a curve of similar shape, in further refutation of cosmic acceleration.

\section{Conclusions}

For any mathematical model of gravitation and cosmology to be superior to general relativity, it must pass the canonical tests of gravitation and provide reasonable solutions to the current problems with general relativity. This paper has made readers aware of Krogdahl's relativity and cosmological model of flat space-time, which appears to achieve these worthy goals.

The focus of this paper has been to show how Krogdahl's relativity provides reasonable solutions to the general relativity problems of the Schwarzschild metric and the infinite singularity, the global cosmic space-time flatness problem, the non-zero cosmological constant problem and the mystery of dark energy. Figure 1 and Figure 2 have provided proof that an expanding flat space-time cosmic model following Krogdahl's relativity fits the tight constraints of current observational data. Finally, two very specific validation predictions concerning the true nature of dark energy are made which would provide further proof that Krogdahl's relativity is superior to general relativity.

\section{References}

[1] Krogdahl, W.S. (2006) Cosmology in Flat Space-Time. arXiv:gr-qc/0402016.

[2] Penrose, R. (1965) Physical Review Letters, 14, 57.

https://doi.org/10.1103/PhysRevLett.14.57

[3] Dicke, R.H. (1970) Gravitation and the Universe. American Philosophical Society. 
[4] Planck Collaboration (2015) XIII. Cosmological Parameters. http://arxiv.org/abs/1502.01589

[5] Nielsen, J.T., et al. (2015) Marginal Evidence for Cosmic Acceleration from Type Ia Supernovae. arXiv:1506.01354v1

[6] Wei, J.-J., et al. (2015) Astronomical Journal, 149, 102. https://doi.org/10.1088/0004-6256/149/3/102

[7] Kipreos, E. (2014) PLOS ONE, 9, e115550, 1-20. https://doi.org/10.1371/journal.pone.0115550

[8] Mostaghel, N. (2016) International Journal of Astronomy and Astrophysics, 6, 122-134. https://doi.org/10.4236/ijaa.2016.61010

[9] Riess, A., et al. (1998) Astrophysical Journal, 473, 1009-1038. https://doi.org/10.1086/300499

[10] Perlmutter, S., et al. (1999) Astrophysical Journal, 517, 565-586. https://doi.org/10.1086/307221

[11] Schmidt, B., et al. (1998) Astrophysical Journal, 507, 46-63. https://doi.org/10.1086/306308

[12] Weinberg, S. (1989) Reviews of Modern Physics, 61, 1-23. https://doi.org/10.1103/RevModPhys.61.1

[13] Carroll, S. (2001) Living Reviews in Relativity, 4, 5-56. https://doi.org/10.12942/lrr-2001-1

[14] Tatum, E.T., Seshavatharam, U.V.S. and Lakshminarayana, S. (2015) International Journal of Astronomy and Astrophysics, 5, 116-124. https://doi.org/10.4236/ijaa.2015.52015

[15] Tatum, E.T., Seshavatharam, U.V.S. and Lakshminarayana, S. (2015) Journal of Applied Physical Science International, 4, 18-26.

[16] Pierseaux, Y. (2014) Journal of Modern Physics, 5, 1725-1732. https://doi.org/10.4236/jmp.2014.516172

[17] Guth, A.H. (1997) The Inflationary Universe. Basic Books, New York.

[18] Perlmutter, S. (2016) Supernova Cosmology Project. Berkeley (US): Lawrence Berkeley National Laboratory. http://www-supernova.lbl.gov

[19] Suzuki, N., et al. (2011) The Hubble Space Telescope Cluster Supernovae Survey: V. Improving the Dark Energy Constraints Above $\mathrm{Z}>1$ and Building an Early-Type-Hosted Supernova Sample. arXiv.org/abs/1105.3470 